\title{
Clinical Features and Risk Factors Associated With 30-Day Mortality in Patients With Pneumonia Caused by Hypervirulent Klebsiella pneumoniae (hvKP)
}

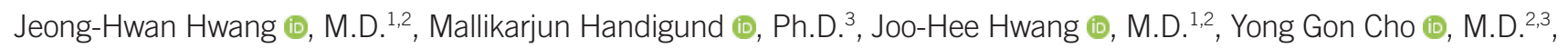

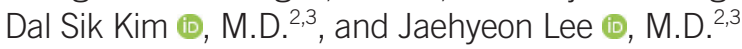

${ }^{1}$ Department of Internal Medicine, Jeonbuk National University Medical School and Hospital, Jeonju, Korea; ${ }^{2}$ Research Institute of Clinical Medicine of Jeonbuk National University-Biomedical Research Institute of Jeonbuk National University Hospital, Jeonju, Korea; ${ }^{3}$ Department of Laboratory Medicine, Jeonbuk National University Medical School and Hospital, Jeonju, Korea

Background: Reports on metastatic or invasive infections by hypervirulent Klebsiella pneumoniae (hvKP) have increased recently. However, the effects of its virulence on clinical course and outcomes in pneumonia patients have rarely been addressed. We assessed and compared the clinical features of hvKp and classic K. pneumoniae (cKP) strains isolated from patients with pneumonia caused by $K$. pneumoniae. We also investigated the effects of virulence factors and the $K$. pneumoniae capsular serotypes $\mathrm{K} 1$ and $\mathrm{K} 2$ on mortality.

Methods: In this retrospective study, we enrolled 91 patients diagnosed as having pneumonia caused by K. pneumoniae and obtained their demographic and clinical data from medical records. We evaluated genes for $\mathrm{K} 1$ and $\mathrm{K} 2$, antimicrobial susceptibility, and the virulence genes $r m p A$, iutA, entB, ybtS, kfu, mrkD, and allS. Strains that possessed $r m p A$ and iutA were defined as hvKP $(N=39)$, while the remaining were classified as $C K P(N=52)$. Odds ratio (OR) for the risk factors associated with 30-day mortality was calculated using the binary logistic regression model.

Results: The 30-day mortality in all patients was $23.1 \%$; it was $17.9 \%(7 / 39)$ in the hvKP group and $26.9 \%(14 / 52)$ in the cKP group $(P=0.315)$. Bacteremia $(O R=38.1 ; 95 \%$ confidence interval $[\mathrm{Cl}], 2.5-570.2)$, altered mental status (OR=8.8; 95\% $\mathrm{Cl}, 1.7-45.0)$, and respiratory rate $>30$ breaths $/ \mathrm{min}(\mathrm{OR}=4.8 ; 95 \% \mathrm{Cl}, 1.2-20.0)$ were independent risk factors for 30-day mortality in all patients.

Conclusions: Our results suggest that hypervirulence determinants do not have a significant effect on 30-day mortality in patients with pneumonia caused by $K$. pneumoniae.

Key Words: Hypervirulence, Klebsiella pneumoniae, Pneumonia, Mortality
Received: December 26, 2019

Revision received: February 18, 2020

Accepted: June 1, 2020

\section{Corresponding author:}

Jaehyeon Lee, M.D., Ph.D.

Department of Laboratory Medicine, Jeonbuk National University Medical

School and Hospital, 20 Geonji-ro, Deokjingu, Jeonju 54907, Korea

Tel: +82-63-250-2693

Fax: +82-63-250-1200

E-mail: laboratorymedicine@gmail.com

\section{INTRODUCTION}

Klebsiella pneumoniae, a leading cause of healthcare-associated infection, is an opportunistic pathogen that causes infection in immunocompromised and hospitalized patients [1]. $\mathrm{K}$. pneumoniae is the second most common cause of community- acquired pneumonia (CAP) in Asia and the most common gramnegative pathogen [2]. In hospital-acquired pneumonia (HAP), including ventilator-associated pneumonia (VAP), K. pneumoniae is the second most common gram-negative pathogen [3].

In the 1980s, a clinical syndrome characterized by endophthalmitis associated with liver abscess due to community-acquired 
K. pneumoniae was described in Taiwan [4]. K. pneumoniae can be classified as classic K. pneumoniae (cKP) or hypervirulent K. pneumoniae (hvKP); hvKP differs from cKP in its clinical and phenotypic characteristics [5, 6]. cKP strains have been associated with infections involving the urinary tract, lungs, abdominal cavity, intravascular regions, surgical sites, and soft tissue and have been identified as the cause of subsequent bacteremia in hospitals and long-term care facilities [6]. In contrast, hvKP strains have been associated with invasive and metastatic infections, including pyogenic liver abscesses, bacteremia, and necrotizing fasciitis, in healthy adults and diabetes patients [7].

At least 78 capsular polysaccharide serotypes exist in K. pneumoniae [6]. For hvKP strains, eight capsular serotypes have been described to date, namely, K1, K2, K5, K16, K20, K54, K57, and KN1 [6]. According to recent reports, the K1 and K2 serotypes are mainly associated with hvKP, and virulence factors, such as $r m p A$, aerobactin, $k f u$, and allS, are more dominant in hvKP than in cKP $[8,9]$. However, few studies have explored the clinical characteristics and 30-day mortality in view of the virulence factors or K1 and K2 serotypes, which are closely related to pneumonia caused by hvKP $[10,11]$. To our knowledge, this is the first study to examine the effects of the $\mathrm{K} 1$ and $\mathrm{K} 2$ serotypes and the virulence genes $r m p A$, iutA, entB, ybtS, $k f u, m r k D$, and allS on 30-day mortality in patients with pneumonia caused by $K$. pneumoniae.

\section{MATERIALS AND METHODS}

\section{Study setting and data collection}

This was a retrospective study conducted at Jeonbuk National University Hospital, Jeonju, Korea. All patients (aged $\geq 19$ years) were diagnosed as having pneumonia caused by $K$. pneumoniae between January 2014 and December 2014. During the study period, a total of 91 patients with pneumonia due to K. pneumoniae were identified (men, 65 [71.4\%]; mean age, 67.7 \pm 14.4 years). All the laboratory tests were performed in routine clinical practice. All samples intentionally collected for this study were cultured in blood agar and MacConkey agar plates in a $35^{\circ} \mathrm{C}$ incubator for 16-24 hours and identified using the Vitek MS system (BioMérieux, Hazelwood, MI, USA). All antimicrobial susceptibility tests (ASTs) were performed using Vitek 2 AST 211 cards (BioMérieux, Marcy-l'Étoile, France) and interpreted using the VITEK 2 identification system, according to the Clinical and Laboratory Standards Institute M100 S16:2016 guidelines [12]. All procedures were performed according to the manufacturer's instructions. After routine practice, all isolates were stored in skim milk in a deep freezer at $-80^{\circ} \mathrm{C}$. When there were multiple strains of $K$. pneumoniae in a respiratory sample (sputum, bronchoalveolar lavage, and endotracheal aspirate), only the first isolated strain was analyzed. We retrospectively reviewed the medical records of the patients and collected the following data: age, sex, vital signs, underlying diseases, type of pneumonia, concomitant bacteremia, clinical signs and symptoms, laboratory findings, antimicrobial used for the episode of pneumonia, and AST results. The primary clinical outcome was 30-day mortality. We selected the associated risk factors on 30-day mortality based on a review of the literature on pneumonia and clinicians' experiences prior to medical records review. The protocol for this study was approved by the Institutional Review Board of Jeonbuk National University Hospital (IRB No. 2017-12-022), which waived the requirement of obtaining informed consent from patients.

\section{Definitions}

HAP was defined as pneumonia occurring $\geq 48$ hours after hospital admission that did not meet all of the healthcare-associated pneumonia (HCAP) criteria [13]. HCAP was defined as pneumonia in patients with more than one of the following risk factors for infection by multidrug-resistant bacteria: hospitalization for two or more days in the past 90 days; residency in a nursing home or long-term care facility; receipt of antimicrobial treatment, chemotherapy, or wound care within 30 days before the current infection; receipt of hemodialysis; and receipt of home wound care [13]. CAP was defined as pneumonia that developed outside the hospital, long-term care facility, or nursing home setting that did not meet the HCAP criteria [13, 14]. hvKP was defined based on positivity for both $r m p A$ and iutA [9], and the remaining cases were classified as CKP. Appropriate antimicrobial treatment was defined as the use of antibiotics with activity against the target pathogen within 24 hours after diagnosis of pneumonia [13].

\section{Microbiological evaluation of virulence genes and capsular serotypes}

We extracted DNA from stored $K$. pneumoniae strains by the boiling method [15], using a DNA extraction buffer (Seegene, Seoul, Korea). Two or three loopfuls of colonies on blood agar plates were transferred into an Eppendorf tube containing $1 \mathrm{~mL}$ of distilled water and $100 \mu \mathrm{L}$ of DNA extraction solution. After vortexing, the solution was boiled at $95^{\circ} \mathrm{C}$ for 20 minutes and centrifuged at $15,000 \times g$ in a microcentrifuge for 10 minutes. The supernatant was used as the template for multiplex PCR to 
detect the $\mathrm{K} 1$ and $\mathrm{K} 2$ capsular serotypes and virulence genes. Primer sets for magA (wzy-like polymerase specific to K1 strains), wzi (the gene specifying the $\mathrm{K} 2$ capsular serotype), and other virulence genes (rmpA, iutA, entB, ybtS, $k f u, m r k D$, and allS) were described previously [16].

\section{Statistical analysis}

Descriptive statistics included frequency analysis (percentages) for categorical variables and mean \pm standard deviation or median (range) for continuous variables. We analyzed categorical variables using chi-square test or Fisher's exact test; we analyzed continuous variables using Student's t-test or Mann-Whitney U-test, as appropriate. $P<0.05$ was considered statistically significant, and all probability values were two-tailed. We performed univariate and multivariate logistic regression analyses to evaluate the risk factors for 30-day mortality in patients with pneumonia caused by $K$. pneumoniae. Variables that showed a significant difference $(P<0.1)$ in the univariate analysis were included in the multivariate analysis. All statistical analyses were conducted with MedCalc Statistical Software ver.19.2.1. (MedCalc Software Ltd., Ostend, Belgium).

\section{RESULTS}

\section{Clinical features of the patients}

The baseline characteristics of the patients are presented in Table 1. hvKP strains were obtained from 39 (42.9\%) patients and cKP strains from $52(57.1 \%)$ patients. In all patients, chronic lung disease ( $\mathrm{N}=44,48.4 \%$ ) was the most common underlying disease, followed by cerebrovascular disease ( $N=39,42.9 \%)$, and CAP, HCAP, and HAP accounted for $40.7 \%(\mathrm{~N}=37), 24.2 \%$ $(\mathrm{N}=22)$, and $37.4 \%(\mathrm{~N}=34)$, respectively. Patients harboring CKP strains had a significantly higher prevalence of malignancy than those harboring hvKP strains (30.8\% vs $12.8 \%, P=0.044$ ).

\section{Microbiological characteristics and antimicrobial susceptibility}

Table 2 compares the frequencies of capsular serotypes and virulence factors between hvKP and cKP strains. The capsular serotypes K1 and K2 accounted for 15.4\% (14/91) and 17.6\% (16/91) of all K. pneumoniae strains, respectively. hvKP strains had a significantly higher prevalence of the $\mathrm{K} 1$ serotype (35.9\% vs $0 \%, P<0.001)$, alls (38.5\% vs $3.8 \%, P<0.001)$, kfu (35.9\% vs $11.5 \%, P=0.005)$, and $y b t S(76.9 \%$ vs $46.2 \%, P=0.003)$ than cKP strains. hvKP strains had a significantly higher median number of virulence factors than $\mathrm{CKP}$ strains $(P<0.001)$.
Table 1. Baseline characteristics of patients with pneumonia caused by Klebsiella pneumoniae

\begin{tabular}{|c|c|c|c|}
\hline Characteristic & $\begin{array}{c}\text { hvKP } \\
(\mathrm{N}=39)\end{array}$ & $\begin{array}{c}\text { cKP } \\
(\mathrm{N}=52)\end{array}$ & $P$ \\
\hline Age (yr) & $68 \pm 14$ & $67 \pm 15$ & 0.681 \\
\hline Males & $27(69.2)$ & $38(73.1)$ & 0.688 \\
\hline \multicolumn{4}{|l|}{ Underlying diseases } \\
\hline Diabetes mellitus & $12(30.8)$ & $14(26.9)$ & 0.688 \\
\hline Malignancy & $5(12.8)$ & $16(30.8)$ & 0.044 \\
\hline Chronic lung disease & $17(43.6)$ & $27(51.9)$ & 0.431 \\
\hline Chronic kidney disease & $12(30.8)$ & $10(19.2)$ & 0.203 \\
\hline Chronic heart disease & $6(15.4)$ & $11(21.2)$ & 0.485 \\
\hline Chronic liver disease & $3(7.7)$ & $4(7.7)$ & 1.000 \\
\hline Cerebrovascular disease & $17(43.6)$ & $22(42.3)$ & 0.903 \\
\hline Charlson's comorbidity index & $6.0 \pm 3.1$ & $6.5 \pm 3.6$ & 0.519 \\
\hline \multicolumn{4}{|l|}{ Type of pneumonia } \\
\hline Community-acquired pneumonia & $17(43.6)$ & $20(34.6)$ & 0.384 \\
\hline Healthcare-associated pneumonia & $7(17.9)$ & $15(28.8)$ & 0.230 \\
\hline Hospital-acquired pneumonia & $15(38.5)$ & $19(36.5)$ & 0.851 \\
\hline \multicolumn{4}{|l|}{ Clinical features at diagnosis of pneumonia } \\
\hline Altered mental status & $16(41.0)$ & $23(44.2)$ & 0.760 \\
\hline Respiratory rate $>30$ breaths $/ \mathrm{min}$ & $5(12.8)$ & $11(21.2)$ & 0.301 \\
\hline Heart rate $>125$ beats $/ \mathrm{min}$ & $9(23.1)$ & $12(23.1)$ & 1.000 \\
\hline Arterial pH $<7.35$ & $9(23.1)$ & $10(19.2)$ & 0.655 \\
\hline Blood urea nitrogen level $>10.71 \mathrm{mmol} / \mathrm{L}$ & $13(33.3)$ & $16(30.8)$ & 0.795 \\
\hline Sodium level $<130 \mathrm{mmol} / \mathrm{L}$ & $5(12.8)$ & $6(11.5)$ & 1.000 \\
\hline Hematocrit $<0.3$ fraction & $19(48.7)$ & $22(42.3)$ & 0.543 \\
\hline Shock at diagnosis & $4(10.3)$ & $8(15.4)$ & 0.474 \\
\hline Bacteremia & $1(2.6)$ & $5(9.6)$ & 0.232 \\
\hline Initial appropriate antibiotics & $32(82.1)$ & $50(96.2)$ & 0.035 \\
\hline 30-day mortality & $7(17.9)$ & $14(26.9)$ & 0.315 \\
\hline
\end{tabular}

Data are presented as mean \pm SD or number (\%).

All 91 K. pneumoniae strains presented uniform susceptibility to ampicillin. There was no significant difference in the antimicrobial susceptibility rate, except for susceptibility to ampicillinsulbactam (Table 2). Although the percentage of extended-spectrum $\beta$-lactamase (ESBL)-producing cKP strains was higher than that of ESBL-producing hvKP strains, the difference was not statistically significant (25.6\% vs $34.6 \%, P=0.359)$.

Risk factors associated with 30-day mortality in patients with pneumonia caused by K. pneumoniae

The overall 30-day mortality in patients with pneumonia caused by K. pneumoniae was $23.1 \%$ (21/91). As shown in Table 3, 
Table 2. Frequencies of capsular serotypes, virulence factors, and antimicrobial susceptibility between hvKP and cKP strains

\begin{tabular}{|c|c|c|c|}
\hline Variable & $\operatorname{hvKP}(\mathrm{N}=39)$ & $\operatorname{cKP}(\mathrm{N}=52)$ & $P$ \\
\hline \multicolumn{4}{|l|}{ Capsular serotype } \\
\hline K1 & $14(35.9)$ & $0(0)$ & $<0.001$ \\
\hline K2 & $10(25.6)$ & $6(11.5)$ & 0.080 \\
\hline Non K1/K2 & $15(38.5)$ & $46(88.5)$ & $<0.001$ \\
\hline \multicolumn{4}{|l|}{ Virulence factor } \\
\hline ent $B$ & $39(100)$ & 49 (94.2) & 0.257 \\
\hline$y b t S$ & $30(76.9)$ & $24(46.2)$ & 0.003 \\
\hline$k f u$ & $14(35.9)$ & $6(11.5)$ & 0.005 \\
\hline$m r k D$ & 38 (97.4) & 49 (94.2) & 0.632 \\
\hline allS & $15(38.5)$ & $2(3.8)$ & $<0.001$ \\
\hline Number of virulence factors & $3(2-5)$ & $3(0-5)$ & $<0.001$ \\
\hline \multicolumn{4}{|l|}{ Antimicrobial susceptability } \\
\hline Amikacin & $34(87.2)$ & $43(82.7)$ & 0.557 \\
\hline Ampicillin-sulbactam & $28(71.8)$ & $25(48.1)$ & 0.023 \\
\hline Aztreonam & $28(71.8)$ & $29(55.8)$ & 0.118 \\
\hline Cefazolin & 27 (69.2) & $27(51.9)$ & 0.096 \\
\hline Cefepime & $28(71.8)$ & $29(55.8)$ & 0.118 \\
\hline Cefotaxime & $28(71.8)$ & $28(53.8)$ & 0.082 \\
\hline Cefoxitin & $30(76.9)$ & $39(75.0)$ & 0.832 \\
\hline Ceftazidime & $28(71.8)$ & $27(51.9)$ & 0.055 \\
\hline Ertapenem & $35(89.7)$ & $44(84.6)$ & 0.474 \\
\hline Gentamicin & 34 (87.2) & $40(76.9)$ & 0.214 \\
\hline Levofloxacin & $29(74.4)$ & $29(55.8)$ & 0.068 \\
\hline Meropenem & $39(100)$ & 48 (92.3) & 0.132 \\
\hline Piperacillin-tazobactam & $29(74.4)$ & $30(57.7)$ & 0.099 \\
\hline Tigecycline & $37(94.9)$ & $45(86.5)$ & 0.291 \\
\hline ESBL & $10(25.6)$ & $18(34.6)$ & 0.359 \\
\hline
\end{tabular}

Data are presented as median (range) or number (\%).

Abbreviations: hvKP, hypervirulent Klebsiella pneumoniae; cKP, classic $K$. pneumoniae; ESBL, extended-spectrum $\beta$-lactamase; NA, not available.

bacteremia $(P=0.002)$, shock at diagnosis $(P=0.005)$, altered mental status $(P<0.001)$, high respiratory rate $(P=0.002)$, heart rates $>125$ beats $/ \mathrm{min}(P<0.001)$, arterial $\mathrm{pH}<7.35(P=0.001)$, blood urea nitrogen $>10.71 \mathrm{mmol} / \mathrm{L}(P=0.001)$, and hematocrit $<0.3$ fraction $(P=0.023)$ were significantly associated with 30-day mortality. Multivariate analysis showed that bacteremia, altered mental status, and respiratory rates $>30$ breaths/min were independent risk factors for 30-day mortality in all patients (Tables 3 and 4).
Table 3. Clinical variables associated with 30-day mortality in patients with pneumonia caused by Klebsiella pneumoniae

\begin{tabular}{|c|c|c|c|}
\hline Characteristic & $\begin{array}{l}\text { Death } \\
(\mathrm{N}=21)\end{array}$ & $\begin{array}{l}\text { Survival } \\
(\mathrm{N}=70)\end{array}$ & $P$ \\
\hline Age (yr) & $68 \pm 14$ & $68 \pm 15$ & 0.961 \\
\hline Male sex & $17(81.0)$ & $48(68.6)$ & 0.271 \\
\hline \multicolumn{4}{|l|}{ Underlying diseases } \\
\hline Diabetes mellitus & $5(23.8)$ & $21(30.0)$ & 0.582 \\
\hline Malignancy & $7(33.3)$ & $14(20.0)$ & 0.241 \\
\hline Chronic lung disease & $10(47.6)$ & $34(48.6)$ & 0.939 \\
\hline Chronic kidney disease & $6(28.6)$ & $16(22.9)$ & 0.592 \\
\hline Chronic heart disease & $3(14.3)$ & $14(20.0)$ & 0.753 \\
\hline Chronic liver disease & $2(9.5)$ & $5(7.1)$ & 0.660 \\
\hline Cerebrovascular disease & $9(42.9)$ & $30(42.9)$ & 1.000 \\
\hline Charlson's comorbidity index & $7.0 \pm 3.3$ & $6.0 \pm 3.4$ & 0.253 \\
\hline \multicolumn{4}{|l|}{ Type of pneumonia } \\
\hline Community-acquired pneumonia & $5(23.8)$ & $30(42.9)$ & 0.116 \\
\hline Healthcare-associated pneumonia & $6(28.6)$ & 16 (22.9) & 0.592 \\
\hline Hospital-acquired pneumonia & $10(47.6)$ & $24(34.3)$ & 0.268 \\
\hline \multicolumn{4}{|l|}{ Serotype } \\
\hline K1 & $2(9.5)$ & $12(17.1)$ & 0.508 \\
\hline K2 & $3(14.3)$ & $13(18.6)$ & 0.756 \\
\hline Non $\mathrm{K} 1 / \mathrm{K} 2$ & 16 (76.2) & $45(64.3)$ & 0.309 \\
\hline \multicolumn{4}{|l|}{ Virulence factor } \\
\hline entB & $20(95.2)$ & $68(97.1)$ & 0.549 \\
\hline$y b t S$ & $13(61.9)$ & $41(58.6)$ & 0.785 \\
\hline$k f u$ & $5(23.8)$ & $15(21.4)$ & 0.773 \\
\hline$m r k D$ & 20 (95.2) & $67(95.7)$ & 1.000 \\
\hline alls & $3(14.3)$ & $14(20.0)$ & 0.753 \\
\hline Number of virulence factors & $3(2-5)$ & $3(0-5)$ & 0.871 \\
\hline Hypervirulent K. pneumoniae & $7(33.3)$ & $32(45.7)$ & 0.315 \\
\hline \multicolumn{4}{|l|}{ Clinical features at diagnosis of pneumonia } \\
\hline Altered mental status & $17(81.0)$ & $22(31.4)$ & $<0.001$ \\
\hline Respiratory rate $>30$ breaths/min & $9(42.9)$ & $7(10.0)$ & 0.002 \\
\hline Heart rate $>125$ beats $/ \mathrm{min}$ & $12(57.1)$ & $9(12.9)$ & $<0.001$ \\
\hline Arterial pH $<7.35$ & $10(47.6)$ & 9 (12.9) & 0.001 \\
\hline Blood urea nitrogen level >10.71 mmol/L & $13(61.9)$ & $16(22.9)$ & 0.001 \\
\hline Sodium level $<130 \mathrm{mmol} / \mathrm{L}$ & $5(23.8)$ & $6(8.6)$ & 0.118 \\
\hline Hematocrit $<0.3$ fraction & $14(66.7)$ & $27(38.6)$ & 0.023 \\
\hline Shock at diagnosis & $7(33.3)$ & $5(7.1)$ & 0.005 \\
\hline Bacteremia & $5(23.8)$ & $1(1.4)$ & 0.002 \\
\hline Initial appropriate antibiotics & $18(85.7)$ & $64(91.4)$ & 0.426 \\
\hline
\end{tabular}

Data are presented as mean \pm SD or median (range) or number (\%). 
Table 4. Multivariate analysis of 30-day mortality in the patients with pneumonia caused by Klebsiella pneumoniae

\begin{tabular}{|c|c|c|c|c|}
\hline \multirow{2}{*}{ Variable } & \multicolumn{2}{|c|}{ Univariate analysis } & \multicolumn{2}{|c|}{ Multivariate analysis } \\
\hline & OR (95\% Cl) & $P$ & OR (95\% Cl) & $P$ \\
\hline Bacteremia & $21.6(2.4-197.5)$ & 0.007 & $38.1(2.5-570.2)$ & 0.008 \\
\hline Altered mental status & $9.3(2.8-30.8)$ & $<0.001$ & $8.8(1.7-45.0)$ & $0.00 s$ \\
\hline Respiratory rate $>30$ breaths $/$ min & $6.8(2.1-21.6)$ & 0.001 & $4.8(1.2-20.0)$ & 0.031 \\
\hline Blood urea nitrogen level $>10.71 \mathrm{mmol} / \mathrm{L}$ & $5.5(1.9-15.6)$ & 0.001 & & \\
\hline
\end{tabular}

Abbreviations: OR, odds ratio; $\mathrm{Cl}$, confidence interval.

\section{DISCUSSION}

This study aimed to assess and compare the clinical features of hvKP and cKP strains isolated from patients with pneumonia caused by $K$. pneumoniae and to investigate the effect of virulence factors and the K1 and K2 serotypes on mortality. This study is significant because, unlike other studies [10, 11], it compared hvKP and cKP considering all types of pneumonia caused by K. pneumoniae, including CAP, HCAP, and HAP. Our results demonstrated that the presence of virulence factors and the $\mathrm{K} 1$ and $\mathrm{K} 2$ serotypes did not affect mortality, whereas bacteremia, altered mental status, and respiratory rates $>30$ breaths/ min were independent risk factors for mortality.

There were no significant risk factors associated with hvKP infection compared with CKP infection. Malignancy was associated with a significantly higher risk for CKP infection, which is consistent with the fact that hospitalized cancer patients are highly susceptible to CKP infection [7]. CKP is the primary strain of nosocomial infection, unlike hvKP, which is associated with CAP [7]. However, we found no significant difference between hvKP and CKP in CAP, HCAP, and HAP patients. In line with this finding, a study conducted in China revealed no difference between hvKP and cKP in HAP caused by K. pneumoniae [17].

Studies in Asia, Europe, and America showed that K1 and K2 have a close relationship with hvKP [5]. In our study, K1 and K2 serotypes were present in $61.5 \%$ of hvKP; this frequency was significantly higher than that of CKP. In two recent studies on VAP caused by $K$. pneumoniae, the prevalence rates of $K 1$ and K2 serotypes in hvKP were $70.6 \%$ and $64.3 \%$, respectively. Further, in bacteremic CAP, the prevalence rate of the $\mathrm{K} 1$ and $\mathrm{K} 2$ serotypes in hvKP was $53.1 \%$, which was higher than that in cKP $(46.9 \%)[10,14,18]$. The prevalence of $K 1$ and K2 is high in hvKP because strains of these serotypes are more resistant to phagocytosis and intracellular elimination by macrophages and neutrophils than are strains of other serotypes $[19,20]$. In addition, a study on VAP reported that the number of virulence fac- tors associated with hvKP is significantly higher than that associated with CKP [18].

In this study, the 30-day mortality in all patients with pneumonia due to $K$. pneumoniae was $23.1 \%$ (21/91). It was $14.3 \%$ (5/35) for CAP, 27.3\% (6/22) for HCAP, 29.4\% (10/34) for HAP, and $19.3 \%(11 / 57)$ for community-onset pneumonia (CAP and HCAP). The mortality associated with community-onset pneumonia caused by $K$. pneumoniae in this study was lower than that in Taiwan (CAP, 25.5\%; HCAP, 34.1\%; and community-onset pneumonia, 29.3\%) [11]. However, in this study, the mortality due to K. pneumoniae-associated CAP was higher than the previous data (7.3\%) due to total pathogens [2]. The mortality due to HAP was similar to the finding (25-58\%) reported in a previous study [3].

There was no significant difference in mortality associated with the hvKP and cKP strains in our study. Moreover, the K1 and K2 serotypes and the virulence genes (rmpA, iutA, entB, ybtS, kfu, $m r k D$, and allS) were not independent risk factors for mortality. In univariate analysis, the unadjusted odds ratio (OR) of hvKP mortality was 0.6 (95\% confidence interval [Cl], 0.2-1.7). After adjustment for confounding variables in multivariate logistic regression analysis, the adjusted OR of hvKP was $0.8(95 \% \mathrm{Cl}$, 0.2-2.9) (data not shown). Therefore, hvKP was not associated with mortality. Similar results have been reported previously [10, $11,14]$. There was no significant difference in mortality between hvKP- and cKP-associated bloodstream infections [21, 22]. However, the pks gene cluster has been found to affect mortality in bloodstream infections caused by K. pneumoniae, and further studies on other virulence factors that affect mortality and on the relationships between virulence factors that contribute to mortality are warranted [23].

ASTs revealed no significant difference between the hvKP and cKP strains, except for susceptibility to ampicillin-sulbactam. In previous studies, resistance to third-generation cephalosporins in hvKP-associated VAP were 0-10\% [18], 14.3\% [24], and $30-40 \%$ [10]. In this study, the resistance rate of hvKP to cefo- 
taxime was $29.2 \%$. However, antibiotic resistance in hvKP may need monitoring as the prevalence of antibiotic-resistant-hvKP is increasing gradually, and early antibiotic administration is important for survival of pneumonia patients [25-27].

Our study had several limitations. First, it was a retrospective, single center study conducted in a small population. However, it is larger than the populations in previous studies on pneumonia due to $K$. pneumoniae [16, 17, 24]. Second, we could not evaluate all the risk factors that would be accessible in an observational study. Third, a standardized definition of hvKP has not yet been established. Although definitions of hvKP differ across studies, iuc and either rmpA or rmpA2 should be considered a key virulence factor of hVKP. In this study, of the 41 strains positive for iutA, 39 harbored rmpA. However, we did not analyze the presence of rmpA2 in two strains. Finally, we investigated only some virulence factors and tested none of the virulence factors in an animal study. We cannot exclude the possibility of inconsistencies between virulence experiments conducted by different groups.

In conclusion, to our knowledge, this is the first study comparing the influence of hvKp and cKP on the mortality in patients with pneumonia caused by $K$. pneumoniae. Our study is of value in investigating the relationship between bacterial virulence and the clinical outcome of pneumonia. We report that hvKP strains do not have a clinically significant effect on mortality in these patients. Future studies are required to confirm the interactions between related virulence factors and the role of other virulence factors in the clinical outcomes of pneumonia caused by K. pneumoniae.

\section{ACKNOWLEDGMENTS}

We thank Ms. Hye-Ran Kim for assistance with the experiments.

\section{AUTHOR CONTRIBUTIONS}

Jeong- $\mathrm{H} \mathrm{H}$ and $\mathrm{JHL}$ designed the study, analyzed the data, and wrote the manuscript. Jeong- $\mathrm{H} \mathrm{H}$ and Joo- $\mathrm{H} \mathrm{H}$ contributed to data collection and ensuring the integrity of the data. JHL designed the microbiological tests, and $\mathrm{MH}$ and $\mathrm{JHL}$ conducted the microbiological tests. Jeong- $\mathrm{H} \mathrm{H}$ contributed to statistical data analysis and interpretation. Jeong- $\mathrm{H} \mathrm{H}$ and $\mathrm{JHL}$ were the major contributors to the study conception and manuscript revision. YGC and DSK contributed to the manuscript revision. All authors have read and approved the final manuscript.

\section{CONFLICTS OF INTEREST}

The authors declare that no conflict of interest exists.

\section{RESEARCH FUNDING}

This paper was supported by funding from the Biomedical Research Institute of Jeonbuk National University Hospital.

\section{ORCID}

Jeong-Hwan Hwang https://orcid.org/0000-0002-2897-2202 Mallikarjun Handigund https://orcid.org/0000-0002-6496-4437 Joo-Hee Hwang Yong Gon Cho

Dal Sik Kim

Jaehyeon Lee https://orcid.org/0000-0002-8616-3411 https://orcid.org/0000-0003-3011-6875 https://orcid.org/0000-0001-7467-2086 https://orcid.org/0000-0003-3211-8903

\section{REFERENCES}

1. Martin RM and Bachman MA. Colonization, infection, and the accessory genome of Klebsiella pneumoniae. Front Cell Infect Microbiol 2018; 8:4.

2. Song JH, Oh WS, Kang Cl, Chung DR, Peck KR, Ko KS, et al. Epidemiology and clinical outcomes of community-acquired pneumonia in adult patients in Asian countries: a prospective study by the Asian network for surveillance of resistant pathogens. Int J Antimicrob Agents 2008; 31:107-14

3. Chawla R. Epidemiology, etiology, and diagnosis of hospital-acquired pneumonia and ventilator-associated pneumonia in Asian countries. Am J Infect Control 2008;36(S4):S93-100.

4. Liu YC, Cheng DL, Lin CL. Klebsiella pneumoniae liver abscess associated with septic endophthalmitis. Arch Intern Med 1986;146:1913-6.

5. Lee CR, Lee JH, Park KS, Jeon JH, Kim YB, Cha CJ, et al. Antimicrobial resistance of hypervirulent Klebsiella pneumoniae: epidemiology, hypervirulence-associated determinants, and resistance mechanisms. Front Cell Infect Microbiol 2017;7:483.

6. Shon AS, Bajwa RP, Russo TA. Hypervirulent (hypermucoviscous) Klebsiella pneumoniae: a new and dangerous breed. Virulence 2013;4:10718.

7. Paczosa MK and Mecsas J. Klebsiella pneumoniae: going on the offense with a strong defense. Microbiol Mol Biol Rev 2016;80:629-61.

8. Wu H, Li D, Zhou H, Sun Y, Guo L, Shen D. Bacteremia and other body site infection caused by hypervirulent and classic Klebsiella pneumoniae. Microb Pathog 2017;104:254-62.

9. Russo TA and Marr CM. Hypervirulent Klebsiella pneumoniae. Clin Microbiol Rev 2019;32:e00001-19.

10. Liu C and Guo J. Characteristics of ventilator-associated pneumonia due to hypervirulent Klebsiella pneumoniae genotype in genetic background for the elderly in two tertiary hospitals in China. Antimicrob Resist Infect Control 2018;7:95.

11. Lin YT, Wang YP, Wang FD, Fung CP. Community-onset Klebsiella pneumoniae pneumonia in Taiwan: clinical features of the disease and asso- 
ciated microbiological characteristics of isolates from pneumonia and nasopharynx. Front Microbiol 2015;9:122.

12. CLSI. Performance standards for antimicrobial susceptibility testing. 28th ed. CLSI supplement M100. Wayne, PA: Clinical and Laboratory Standards Institute, 2018.

13. American Thoracic Society and Infectious Diseases Society of America. Guidelines for the management of adults with hospital-acquired, ventilator-associated, and healthcare-associated pneumonia. Am J Respir Crit Care Med 2005;171:388-416.

14. Lin YT, Jeng YY, Chen TL, Fung CP. Bacteremic community-acquired pneumonia due to Klebsiella pneumoniae: clinical and microbiological characteristics in Taiwan, 2001-2008. BMC Infect Dis 2010;10:307.

15. Ivanov IG and Bachvarov DR. Determination of plasmid copy number by the "boiling" method. Anal Biochem 1987;165:137-41.

16. Compain F, Babosan A, Brisse S, Genel N, Audo J, Ailloud F, et al. Multiplex PCR for detection of seven virulence factors and K1/K2 capsular serotypes of Klebsiella pneumoniae. J Clin Microbiol 2014;52:4377-80.

17. Zhang Y, Zhao C, Wang Q, Wang X, Chen H, Li H, et al. High prevalence of hypervirulent Klebsiella pneumoniae infection in China: geographic distribution, clinical characteristics, and antimicrobial resistance. Antimicrob Agents Chemother 2016;60:6115-20.

18. Yan Q, Zhou M, Zou M, Liu WE. Hypervirulent Klebsiella pneumoniae induced ventilator-associated pneumonia in mechanically ventilated patients in China. Eur J Clin Microbiol Infect Dis 2016;35:387-96.

19. Yeh KM, Kurup A, Siu LK, Koh YL, Fung CP, Lin JC, et al. Capsular serotype $\mathrm{K} 1$ or $\mathrm{K} 2$, rather than magA and $\mathrm{rmpA}$, is a major virulence determinant for Klebsiella pneumoniae liver abscess in Singapore and Taiwan. J Clin Microbiol 2007;45:466-71.
20. Lee CH, Chang CC, Liu JW, Chen RF, Yang KD. Sialic acid involved in hypermucoviscosity phenotype of Klebsiella pneumoniae and associated with resistance to neutrophil phagocytosis. Virulence 2014;5:673-9.

21. Li J, Ren J, Wang W, Wang G, Gu G, Wu X, et al. Risk factors and clinical outcomes of hypervirulent Klebsiella pneumoniae induced bloodstream infections. Eur J Clin Microbiol Infect Dis 2018;37:679-89.

22. Yu WL, Lee MF, Chen CC, Tang HJ, Ho CH, Chuang YC. Impacts of hypervirulence determinants on clinical features and outcomes of bacteremia caused by extended-spectrum $\beta$-lactamase-producing Klebsiella pneumoniae. Microb Drug Resist 2017;23:376-83.

23. Kim D, Park BY, Choi MH, Yoon EJ, Lee H, Lee KJ, et al. Antimicrobial resistance and virulence factors of Klebsiella pneumoniae affecting 30 day mortality in patients with bloodstream infection. J Antimicrob Chemother 2019;74:190-9.

24. Guo S, Xu J, Wei Y, Xu J, Li Y, Xue R. Clinical and molecular characteristics of Klebsiella pneumoniae ventilator-associated pneumonia in mainland China. BMC Infect Dis 2016;16:608.

25. Li W, Sun G, Yu Y, Li N, Chen M, Jin R, et al. Increasing occurrence of antimicrobial-resistant hypervirulent (hypermucoviscous) Klebsiella pneumoniae isolates in China. Clin Infect Dis 2014;58:225-32.

26. Liu Z, Gu Y, Li X, Liu Y, Ye Y, Guan S, et al. Identification and characterization of NDM-1-producing hypervirulent (hypermucoviscous) Klebsiella pneumoniae in China. Ann Lab Med 2019;39:167-75.

27. Palmieri M, Schicklin S, Pelegrin AC, Chatellier S, Franceschi C, Mirande $\mathrm{C}$, et al. Phenotypic and genomic characterization of AmpC-producing Klebsiella pneumoniae from Korea. Ann Lab Med 2018;38:36770 . 